\title{
RELACIÓN ENTRE EL TAMAÑO DE LOS MACROQUISTES DE Sarcocystis aucheniae Y SU VIABILIDAD EN Canis familiaris
}

\author{
RELATIONSHIP BETWEEN THE SIZE OF MACROCYSTS OF SARCOCYSISTIS \\ AUCHENIAE AND ITS VIABILITY IN CANIS FAMILIARIS
}
Rossmery Cornejo B. ${ }^{1}$, Amanda Chávez V. ${ }^{2}$, Victor Leyva V. ${ }^{3}$, Nestor Falcón P. ${ }^{4}$, Susan Panez L. ${ }^{5}$ y Daniel Ticona S. ${ }^{5}$

\section{RESUMEN}

El objetivo del estudio fue determinar si el tamaño de los macroquistes de Sarcocystis aucheniae afectan la viabilidad y potencial biótico en los caninos domésticos. Se utilizaron 24 cachorros cruzados de 2.5 meses de edad, con pesos promedio de $2.6 \mathrm{~kg}$., debidamente desparasitados y alimentados con una dieta casera exenta de carnes. Se obtuvo macrosquistes de $S$. aucheniae del cuello de alpacas y llamas en un camal local, clasificándolos en macroquistes grandes (>5 mm) y pequeños $(1-3 \mathrm{~mm})$. Diez cachorros fueron oralmente infectados con 500 macroquistes grandes (MG) y 12 cachorros con 500 macroquistes pequeños (MP), en ambos casos por dos días consecutivos. Además, 6 perros quedaron sin infectar como grupo control. Se recolectó diariamente muestras fecales a partir del $8^{\circ}$ día post-infección y por un periodo de 22 días. Las muestras se analizaron por el método de flotación con solución de Sheather para determinar la presencia de ooquistes o esporoquistes de Sarcocystis sp. y el método de Stoll modificado para cuantificar su número por gramo de heces. Los resultados mostraron que animales infectados con MP presentaron una carga de esporoquistes 3.6 veces superior que aquellos infectados con MG; así mismo, el periodo prepatente promedio fue de 16.5 y 11.5 para los MG y MP, respectivamente.

Palabras clave: sarcocistiosis, macroquistes, infección, perro, potencial biótico

\section{Abstract}

The objective of the study was to determine weather the size of Sarcocystis aucheniae macrocysts affect its viability and biotic potential in domestic canines. A total of 26 crossbred puppies, 2.5 month of age, $2.6 \mathrm{~kg}$ of body weight, were used. Puppies were dewormed and fed with a diet extent of meat. Macrocysts of S. aucheniae were obtained from alpaca and llama's neck in the local slaughterhouse. Cysts were classified according

1 Práctica privada

${ }^{2}$ Laboratorio de Microbiología y Parasitología Veterinaria, FMV-UNMSM

E-mail: a_chavez_g@hotmail.com

${ }^{3}$ Laboratorio de Reproducción Animal, FMV-UNMSM

${ }^{4}$ Laboratorio de Medicina Veterinaria Preventiva, FMV-UNMSM

${ }^{5}$ Estación Experimental del Centro de Investigación IVITA-Maranganí, FMV-UNMSM 
to size in large (>5 mm) and small (1-3 mm) cysts. Ten puppies were orally infected with 500 large macrocysts (MG) and 12 puppies with 500 small macrocysts (MP), for 2 consecutive days, whereas 6 puppies were kept as controls. Fecal samples were daily collected and analyzed by the flotation method using the Sheather solution to determine the presence of oocysts and sporocysts of Sarcocystis sp. and the modified Stoll method to quantify the number of sporocysts per gram of feces. The results showed that the burden of sporocysts was 3.6 times more in puppies infected with MP than in those infected with MG. Furthermore, the average prepatent period was 16.5 and 11.5 days for MG and MP respectively.

Key words: sarcocystosis, macrocysts, infection, dog, biotic potential

INTRODUCCIÓN

La actividad pecuaria de las comunidades alto andinas está destinada mayormente a la crianza de camélidos sudamericanos (CSA), siendo ésta una actividad de gran importancia social, económica y científica (Carpio, 1991). Dentro de los CSA, la alpaca constituye la especie más atractiva debido al valor comercial de su fibra y la cada vez más creciente demanda de su carne. Lamentablemente, la venta de carne se ve frecuentemente afectada por la presencia de macroquistes de Sarcocystis aucheniae y de microquistes de Sarcocystis lamacanis, causando serias pérdidas económicas a los productores y campesinos de la zona altoandina del Perú (Leguía, 1991).

El S. aucheniae ocasiona quistes macroscópicos de 0.1 a $1 \mathrm{~cm}$ de largo, de un color blanco con apariencia a un grano de arroz compacto, y tienden a crecer lentamente en las fibras musculares esqueléticas. Por el contrario, S. lamacanis produce quistes microscópicos que se desarrollan más rápidamente y con mayor capacidad infectiva, tendiendo a localizarse principalmente en la musculatura cardiaca (Leguía et al., 1990; La Perle et al., 1999). El aspecto desagradable de la carne infectada ocasiona el decomiso de las carcasas en los camales (Alva et al., 1980), con pérdidas anuales estimadas en US\$300,000.00 (Leguía, 1991), convirtiéndose en el principal problema que limita el consumo y la aceptación de la carne a nivel de los mercados (Vilca, 1991).
El ciclo de vida de la sarcocistiosis en CSA es indirecto, donde los cánidos constituyen los únicos hospederos definitivos que se infectan al consumir carne parasitada. En el intestino delgado desarrollan la fase sexual, liberando los ooquistes y esporoquistes infectivos al medio ambiente, los que al ser consumidos por las alpacas y llamas desarrollan la fase asexual en sus tejidos, dando lugar a los quistes macroscópicos y microscópicos (Leguía y Casas, 1999). Infecciones experimentales en perros con macroquistes y microquistes de Sarcocystis de alpacas y llamas (Leguía et al., 1989) evidenciaron periodos prepatentes diferentes. Así, perros infectados con macroquistes presentaron periodos prepatentes de 11-20 días, mientras que con microquistes fueron de 9-14 días, siendo sus periodos patentes de 20-40 y de 60-72 días, respectivamente.

Se viene desarrollando estudios tendientes a controlar el Sarcocystis sp. mediante la utilización de drogas como el ponazuril (Lindsay et al., 2000; Franklin et al., 2003). Así mismo, se está evaluando vacunas para prevenir la sarcocistiosis en alpacas (A. Hung, comunicación personal). Actualmente, la técnica de ELISA es uno de los métodos de laboratorio más utilizados para identificar la presencia de Sarcocystis sp., aunque este método no discrimina entre las especies de S. aucheniae y S. lamacanis, debido a una reacción inmune cruzada entre ellas (Sam, 1988), probablemente debido a la diversidad de antígenos que están presentes durante el desarrollo del parásito. Esta situación conlleva a que la inspección sanitaria de la carne 
es el único método de diagnóstico eficaz y preciso que se cuenta para tal fin, por lo que el presente estudio tuvo por objetivo determinar si el tamaño de los macroquistes de $S$. aucheniae afectan su viabilidad y potencial biótico en caninos domésticos.

\section{Materiales y Métodos}

\section{Ubicación del estudio}

El estudio se llevó a cabo en la Estación Experimental del Centro de Investigaciones IVITA-Maranganí, ubicada a 3,813 msnm, en el distrito de Maranganí, provincia de Canchis, Cusco. La temperatura ambiental máxima varía entre 13 y $14^{\circ} \mathrm{C}$ y la mínima entre -5 y $2{ }^{\circ} \mathrm{C}$, con un promedio de precipitación anual de $953 \mathrm{~mm}$. El estudio se realizó entre febrero y abril del 2006, durante la época lluviosa e inicio de seca.

\section{Macroquistes de $S$. aucheniae}

Se adquirió carne fresca de alpacas y llamas del camal de Ñuñoa, especialmente de la zona del cuello, a fin de obtener macroquistes de $S$. aucheniae. Los quistes fueron clasificados según su tamaño en macroquistes grandes (MG) si fueron mayores de $5 \mathrm{~mm}$ y macroquistes pequeños (MP) cuando midieron entre 1 y $3 \mathrm{~mm}$.

\section{Procedimiento experimental}

Se utilizaron 26 cachorros cruzados de ambos sexos, con edades de 45-60 días y peso promedio de $2.6 \mathrm{~kg}$. Los animales fueron desparasitados y vacunados contra parvovirus y distemper en la etapa pre-experimental; así mismo, fueron alimentados dos veces al día con una dieta casera exenta de carnes rojas y recibieron agua ad libitum. Se alojaron en casetas individuales (caniles de madera) de 1 x 0.7 x $0.9 \mathrm{~m}$, que fueron flameadas y desinfectadas con lejía y formol al $20 \%$ antes de ser usadas. El piso de las jaulas fue cubierto con papel periódico, que era cambiado dos veces al día.
Se trabajó con un grupo control no infectado de 4 cachorros y dos grupos infectados de 10 y 12 cachorros cada uno, que fueron infectados oralmente durante dos días consecutivos con una dosis de 500 macroquistes MG y MP, respectivamente. A partir del $8^{\circ}$ día post-infección se colectó muestras fecales diarias por un periodo de 22 días. Se determinó la presencia de ooquistes y esporoquistes en las heces mediante el método de concentración por flotación con solución azucarada (Sheather). Posteriormente, las muestras positivas fueron cuantificadas mediante el método de Stoll modificado, para determinar el número de esporoquistes por gramo de heces.

\section{Interpretación y análisis estadístico}

La viabilidad de los macroquistes de $S$. aucheniae en los caninos se estableció a través de la observación de ooquistes y esporoquistes en heces. Además, se determinó el periodo prepatente (ppp) en días. $\mathrm{La}$ diferencia entre el número de esporoquistes eliminados por efecto del tamaño de macroquiste se evaluó mediante la prueba de U de Mann Withney y la asociación entre la mortalidad de los animales infectados y el tamaño de macroquistes administrados se determinó mediante la prueba exacta de Fisher. En ambos casos se utilizó el programa estadístico SPSS v. 11.1.

\section{Resultados y Discusión}

Los animales inoculados con macroquistes pequeños de $S$. aucheniae mostraron un ppp más corto (11- 12 días) que aquellos que recibieron macroquistes grandes (15-18 días). No se observó la presencia de ooquistes ni esporoquistes en el grupo control (Cuadro 1).

Los 16.5 días de ppp en promedio obtenido con infección de macroquistes grandes fue similar al hallado por Leguía et al. (1989), quienes encontraron un promedio de 16 días y un rango de 11-20 días, al infectar 
Cuadro 1. Variación del periodo prepatente en caninos infectados con macroquistes grandes y pequeños de S. aucheniae (Maranganí, 2006)

\begin{tabular}{lccc}
\hline \multirow{2}{*}{ Macroquistes } & $\begin{array}{c}\text { Animales } \\
(\mathrm{n})\end{array}$ & \multicolumn{2}{c}{ Periodo prepatente (días) } \\
\cline { 3 - 4 } & 8 & Promedio & Rango \\
\hline Grandes $(>5 \mathrm{~mm})$ & 6 & 16.5 & $15-18$ \\
Pequeños $(1-3 \mathrm{~mm})$ & 4 & 11.5 & $11-12$ \\
Control (no infectado) & & - & - \\
\hline
\end{tabular}

Cuadro 2. Promedio diario de esporoquistes por gramo de heces (eph) en caninos infectados con macroquistes grandes $(>5 \mathrm{~mm})$ y pequeños $(1-3 \mathrm{~mm}) \mathrm{de} S$. aucheniae durante 29 días post-infección (Maranganí, 2006)

\begin{tabular}{ccc}
\hline Días post-infección & $\begin{array}{c}\text { Macroquistes } \\
(\mathrm{epg})\end{array}$ & $\begin{array}{c}\text { grandes }^{1} \\
(\text { epg })\end{array}$ \\
\hline 11 & 0 & 200 \\
12 & 0 & 233 \\
13 & 0 & 3533 \\
14 & 0 & 667 \\
15 & 50 & 933 \\
16 & 225 & 1,133 \\
17 & 400 & 1,333 \\
18 & 229 & 1,240 \\
19 & 286 & 1,400 \\
20 & 343 & 1,560 \\
21 & 457 & 1,880 \\
22 & 543 & 2,800 \\
23 & 686 & 3,600 \\
24 & 771 & 3,760 \\
25 & 886 & 4,280 \\
26 & 1,143 & 3,720 \\
27 & 1,514 & 3,360 \\
28 & 1,543 & 2,880 \\
29 & 1,343 & 2,320 \\
\hline Promedio & 548 & 1,992 \\
\hline $\mathrm{n}=6$ cachorros & & \\
\hline El 100\% presentó esporoquistes en heces & & \\
\hline
\end{tabular}

perros con macroquistes sin que se discriminase el tamaño de los mismos. De la misma forma, el ppp de 11.5 días con infecciones de microquistes pequeños fue similar al prome- dio reportado por Leguía et al. (1989) (11 días) cuando infectaron cachorros con microquistes de $S$. lamacanis, por lo que se podría inferir que tanto microquistes como 


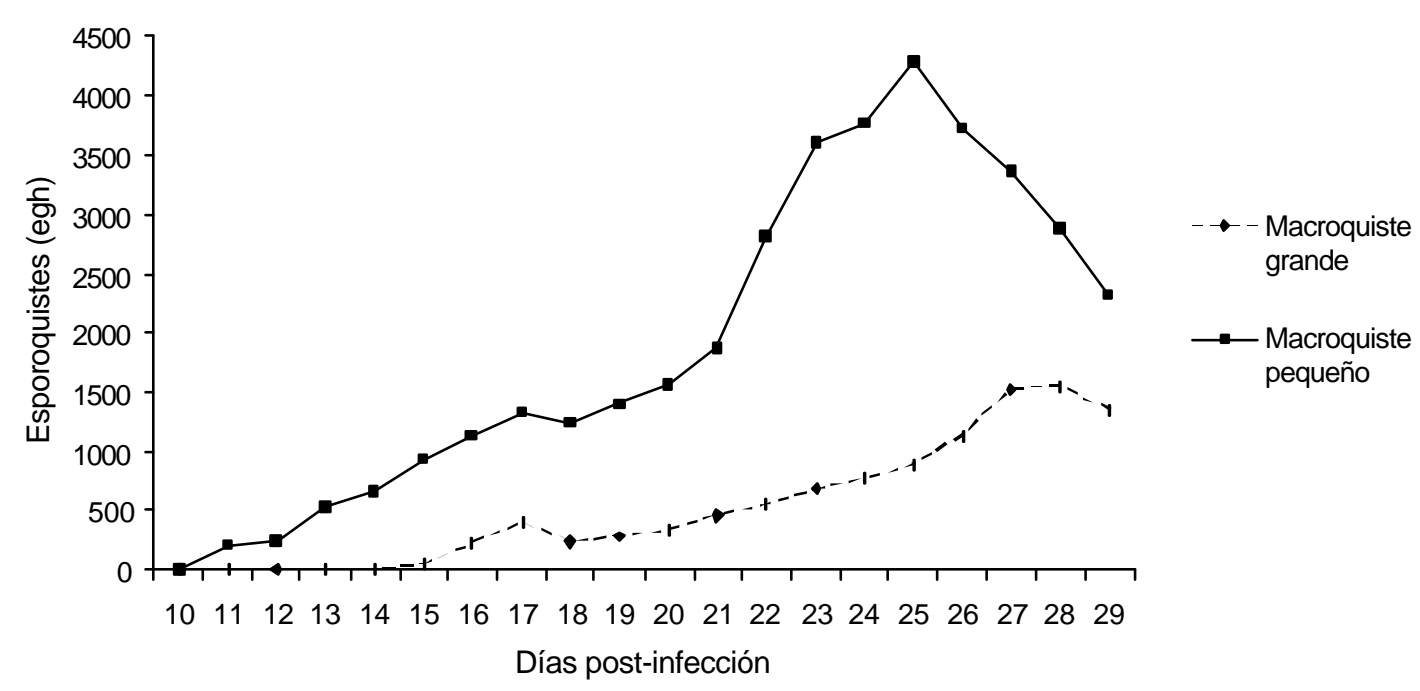

Figura 1. Distribución promedio de esporoquistes por gramo de heces, recuperados en caninos infectados con macroquistes grandes y pequeños de $S$. aucheniae durante 29 días. Maranganí, 2006.

macroquistes menores de $3 \mathrm{~mm}$ presentan ppp similares. Por otro lado, Schnieder et al. (1984) obtuvieron un ppp de 11 días al infectar un perro con macroquistes de $S$. aucheniae provenientes de una llama (Lama glama); sin embargo, este resultado debe tomarse con cautela, toda vez que se trata de un sólo animal y se desconoce si el ppp puede ser diferente si los esporoquistes provienen de una sola especie de CSA.

Estudios realizados con PCR (Hung et $a l ., 2004)$ han permitido identificar $\mathrm{y}$ genotipificar al $S$. aucheniae y al $S$. lamacanis, fijando la diferencia en algunos nucleótidos (SSUR-RNA gen). A pesar de ello, resulta significativo que el presente estudio señale coincidencias biológicas entre las dos especies, por lo que convendría realizar trabajos adicionales tendientes a confirmar dichas diferencias.

En el Cuadro 2 se observa el promedio diario de esporoquistes por gramo de heces (epg) de caninos infectados con macroquistes grandes y pequeños. Se observa una diferencia de 3 días en el inicio de la eliminación de esporoquistes entre ambos tamaños. Así, el
$50 \%$ de cachorros infectados con MP presenta una carga inicial de 200 esporoquistes por gramo de heces (epg) el día 11 post-infección, mientras que sólo el $12.5 \%$ de los animales infectados con MG eliminan 50 epg el día 13. Así mismo, en el mismo lapso de tiempo el grupo infectado con MP eliminó en promedio una carga de 1,992 epg frente al grupo infectado con MG que sólo alcanzó 548 epg, evidenciando que la capacidad biótica de los MP fue 3.6 veces mayor. La mayor eliminación de esporoquistes $(4,280$ hpg $)$ ocurrió en el día 25 post-infección con MP mientras que fue a los 28 días (1,543 hpg) con MG. Los resultados demuestran que infecciones realizadas con MP eliminan un mayor número de esporoquistes en un periodo más corto.

La Fig. 1 muestra la distribución promedio de la carga de esporoquistes durante los 29 días post-infección, observándose un incremento progresivo hasta alcanzar la máxima diferencia en el día 25. Diferencias significativas $(p<0.05)$ en la eliminación de esporoquistes ocurrieron entre los días 11 al 28 post-infección. Al conocer la carga promedio de epg que podrían eliminar cachorros 
infectados con quistes grandes y pequeños de S. aucheniae (Cuadro 2), se podría especular sobre el grado de contaminación de las pasturas que pueden causar cachorros de 2 3 meses de edad, con un peso aproximado de $2.6 \mathrm{~kg}$ y con un promedio diario de evacuación de heces de 78 g. Los cachorros infectados con MG eliminarían una carga total de 120,354 esporoquistes en el pico de su producción (día 28), versus los cachorros infectados con MP quienes eliminarían una carga total de 333,840 esporoquistes en el día 25. Estas cifras revelan que ambos tipos de infecciones ocasionan preocupantes niveles de contaminación en el medio ambiente durante los 40 días que dura su periodo patente.

Así también, se sabe que la administración de una dosis de 40,000 a 160,000 esporoquistes de $S$. aucheniae en alpacas de 4 meses de edad llega a producir cuadros de sarcocistiosis aguda y subaguda, con marcados cambios hematológicos y serológicos en los animales (Sam et al., 1978). Es decir, que sólo bastaría las heces que un cachorro haya evacuado en un día para ocasionar cuadros clínicos en 3 a 8 alpacas. Por otro lado, Leguía et al. (1990) encontraron que al inocular 160,000 esporoquistes de $S$. lamacanis en alpacas de 3 meses de edad produjeron cuadros clínicos entre los 19 y 22 días postinfección, llegando a ocasionar la muerte de los animales entre 3 a 4 días después de la presentación de los signos clínicos. Infecciones experimentales en 13 crías de alpacas de 4 meses de edad utilizando 50,000 esporoquistes de $S$. lamacanis, causaron la muerte al $77 \%$ de los animales dentro de los 2 meses de la infección (A. Chávez, en prensa).

El Cuadro 3 muestra el porcentaje de mortalidad de los cachorros infectados con macroquistes grandes y pequeños de $S$. aucheniae. Se observa que la mortalidad producida por los MP fue casi el doble que la ocasionada por los MG; sin embargo, al evaluar mediante el análisis de Fisher, la asociación entre la cantidad de animales muertos y el tamaño de macroquiste infectante, se demuestra que esa asociación no es significativa.
Cuadro 3. Porcentaje de mortalidad de cachorros infectados con macroquistes grandes $\mathrm{y}$ pequeños de $S$. aucheniae (Maranganí, 2006)

\begin{tabular}{cccc}
\hline \multirow{2}{*}{ Macroquistes } & \multirow{2}{*}{$\begin{array}{c}\text { Total } \\
\text { (n) }\end{array}$} & \multicolumn{2}{c}{ Muertos } \\
\cline { 3 - 4 }$>5 \mathrm{~mm}$ & 10 & 3 & $30^{\mathrm{a}}$ \\
$1-3 \mathrm{~mm}$ & 12 & 7 & $58^{\mathrm{a}}$ \\
\hline Total & 22 & 10 & 45 \\
\hline
\end{tabular}

${ }^{a}$ Sin diferencia estadística

Los animales infectados mostraron signos clínicos caracterizados por pérdida de peso, anorexia, pirexia, palidez de las mucosas, incoordinación y diarreas. Además, aquellos del grupo MG que murieron mostraron diarrea mucosa, mientras que los del grupo MP tuvieron diarrea muco-sanguinolenta, en forma similar a lo reportado por Leguía et al., (1989) cuando infectaron a un cachorro con $200 \mathrm{~g}$ de músculo cardiaco conteniendo microquistes de $S$. lamacanis.

En la actualidad, el $80 \%$ de las alpacas y la casi totalidad de llamas se encuentra en poder de los pequeños productores y las comunidades campesinas (Franco et al., 1998). Es en estas condiciones donde el productor considera al perro no sólo como un fiel amigo, sino además, como apoyo en las faenas de manejo de su ganado. Lamentablemente, las limitadas campañas de educación sanitaria y la poca información sobre el problema han tenido poco impacto y han permitido que se continúe ignorando la trascendencia que tiene el perro como parte de la persistencia de la sarcocistiosis.

\section{Conclusiones}

- La carga de esporoquistes en heces de cachorros infectados con macroquistes pequeños (1-3 $\mathrm{mm}$ ) de $S$. aucheniae fue 
3.6 veces mayor que la carga ocasionada con macroquistes grandes ( $>5 \mathrm{~mm})$.

- Los animales infectados con macroquistes pequeños de $S$. aucheniae tuvieron un periodo prepatente de 11.5 días, mientras que en los infectados con macroquistes grandes de $S$. aucheniae fue de 16.5 días.

- Se encontró una tendencia de mayor mortalidad en caninos infectados con macroquistes pequeños de $S$. aucheniae.

\section{Agradecimiento}

Un agradecimiento especial a INCAGRO, por el apoyo económico en la realización del presente proyecto, enmarcado dentro del subproyecto «Estrategia de manejo genético, sanitario y nutricional en la expresión de la capacidad genética de llamas para producción de carnes». Así también al Dr. Mario García por el apoyo incondicional en la corrección del presente artículo.

\section{Literatura Citada}

1. Alva, J.; M. Rojas; A Nuñez. 1980. Decomisos por parasitosis y su importancia económica en alpacas (Lama pacos). Rev. Inv. Pec. IVITA 5(1): 61.

2. Carpio, M. 1991. Camélidos y socioeconomía andina. En: Producción de rumiantes menores. Cap. 1. C. Novoa; A. Flores (ed). Ed. Rerumen. Lima.

3. Franco, E.; W. García; D. Pezo. 1998. Manual de crianza de llamas. Pub. Tec. IVITA 33: $42 \mathrm{p}$.

4. Franklin, R.P.; R.J. Mackay; K.D. Gillis; S.M. Tanhauser; P.E. Ginn; T.J. Kennedy. 2003. Effect of a single dose of ponazuril on neural infection and clinical disease in Sarcocystis neuronachallenged interferon-gamma knockout mice. Vet. Parasitol. 114: 123-130.
5. Hung, A.; C. Bravo de Rueda; N. Arias; C. Martínez; A.G. Murguía. 2004. Sarcocystis auchenia: Fase 1. Proyecto PROCOM-CONCYTEC. [Internet] [acceso 10 febrero 2007]. Disponible en: http://tumi.lamolina.edu.pe/ estrategia/descarga/archivo2.pdf

6. La Perle, K.M.; F. Silverio; D.E. Anderson; E.A. Blomme. 1999. Dalmeni desease in an aplaca (Lama pacos): Sarcocystosis, eosinophilic, myositis and abortion. J. Comp. Pathol. 121: 287-293.

7. Leguía, G. 1991. The epidemiology and economic impact of llama parasites. Parasit. Today 7: 54-56.

8. Leguía, G.; C. Guerrero; A Chávez; F. Arévalo; R. Sam. 1990. Estudios de la sarcocystiosis en alpacas. Avances sobre Investigación en Salud Animal: Camélidos Sudamericanos 23: 43-46.

9. Leguía, G.; C. Guerrero; R. Sam; A. Chávez. 1989. Infección experimental de perros y gatos con microquistes y macroquistes de sarcocystiosis de alpacas. MV Rev. Cienc. Vet. 5(3):10-13.

10. Leguía, G.; E. Casas. 1999. Enfermedades parasitarias y atlas parasitológico de camélidos sudamericanos. p 23-30. Ed. De Mar. Lima.

11. Lindsay, D.; J. Dubey; T. Kennedy. 2000. Determination of the activity of ponazuril against Sarcocystis neurona in cell cultures .Vet. Parasitol. 92: 165169.

12. Sam, R. 1988. Sarcocystis aucheniae: Caracterización parcial de componentes antigénicos y patología clínica experimental en alpacas. Tesis Doctoral en Ciencias Biológicas. Facultad de Ciencias Biológicas, Univ. Nacional Mayor de San Marcos. Lima. 118 p.

13. Sam, R.; R. Oré; G. Leguía. 1978. Cambios hematológicos y serológicos en alpacas experimentalmente infectadas con Sarcocystis aucheniae. XI Congreso Panamericano de Ciencias Veterinarias. Lima, Perú. 
14. Schnieder, T.; F.J. Kanp; W. Drommer; W. Thiel; M. Rommel. 1984. Fine structure and development of Sarcocystis aucheniae in llamas. Z. Parasitenkd. 70: 451-458.
15. Vilca, M. 1991. Producción, tecnología e higiene de la carne. En: Avances y perspectivas del conocimiento de los camélidos sudamericanos. Fernández Baca, S. (eds). p 387-417. FAO. Santiago, Chile. 